\title{
GCU
}

Glasgow Caledonian

University

University for the Common Good

\section{Barriers and enablers to meticillin-resistant Staphylococcus aureus admission screening in hospitals: a mixed-methods study}

Currie, K.; King, C.; McAloney-Kocaman, K.; Roberts, N. J.; MacDonald, J.; Dickson, A.; Cairns, S.; Khanna, N.; Flowers, P.; Reilly, J.; Price, L.

Published in:

Journal of Hospital Infection

DOI:

10.1016/j.jhin.2018.08.006

Publication date:

2019

Document Version

Author accepted manuscript

Link to publication in ResearchOnline

Citation for published version (Harvard):

Currie, K, King, C, McAloney-Kocaman, K, Roberts, NJ, MacDonald, J, Dickson, A, Cairns, S, Khanna, N,

Flowers, P, Reilly, J \& Price, L 2019, 'Barriers and enablers to meticillin-resistant Staphylococcus aureus admission screening in hospitals: a mixed-methods study', Journal of Hospital Infection, vol. 101, no. 1, pp. 100108. https://doi.org/10.1016/j.jhin.2018.08.006

\section{General rights}

Copyright and moral rights for the publications made accessible in the public portal are retained by the authors and/or other copyright owners and it is a condition of accessing publications that users recognise and abide by the legal requirements associated with these rights.

Take down policy

If you believe that this document breaches copyright please view our takedown policy at https://edshare.gcu.ac.uk/id/eprint/5179 for details of how to contact us. 
Title: The AMR-BESH Study: A mixed methods study of barriers and enablers to MRSA admission screening in hospitals

\section{Authors:}

Currie $\mathrm{K}^{1}$; King $\mathrm{C}^{1}$; McAloney-Kocaman; $\mathrm{K}^{1}$; Roberts $\mathrm{NJ}^{1}$; MacDonald $\mathrm{J}^{1}$; Dickson, $\mathrm{A}^{1}$; Cairns $\mathrm{S}^{2}$; Khanna $\mathrm{N}^{3}$; Flowers $\mathrm{P}^{1}$; Reilly $\mathrm{J}^{1 / 2}$; Price $\mathrm{L}^{1}$.

${ }^{1}$ Safeguarding Health through Infection Prevention [SHIP] Research Group.

Glasgow Caledonian University.

${ }^{2}$ NHS Health Protection Scotland

${ }^{3}$ NHS Greater Glasgow \& Clyde

Corresponding Author: Kay Currie, School of Health \& Life Sciences, Glasgow Caledonian University, Cowcaddens, Glasgow, G4 OBA. Tel: 01413313472 E-mail: k.currie@gcu.ac.uk

Key words: $\quad$ AMR; MRSA; screening; compliance; Theoretical Domains Framework; Normalisation Process Theory

Acknowledgements: This study was funded by NHS Health Protection Scotland and the Scottish Infection Research Network and supported by the Scottish Healthcare Associated Infection Prevention Institute (SHAIPI). We would also like to acknowledge the contribution of the Project Advisory Group and NHS Scotland staff in supporting the conduct of this study.

There are no conflicts of interest to report. 


\section{Summary}

Background: To reduce the risk of transmission of Meticillin resistant Staphylococcus aureus (MRSA), international guidelines recommend admission screening to identify hospital patients at risk of colonisation. However, routine monitoring indicates that optimum screening compliance levels are not always achieved. In order to enhance compliance, we must better understand those factors which influence staff screening behaviours.

Aim: To identify factors which influence staff compliance with hospital MRSA screening policy.

Methods: A sequential 2-stage mixed methods design applied constructs from Normalisation Process Theory and the Theoretical Domains Framework to guide data collection and analysis. Initial qualitative findings informed subsequent development of a national cross-sectional survey of nursing staff $(n=450)$. Multiple regression modelling identified which barriers and enablers' best predict staff compliance.

Findings: Three factors were significant in predicting optimum (>90\%) compliance with MRSA screening: having MRSA screening routinized within the admission process; category of clinical area; feedback of MRSA screening compliance within the clinical area. Integration of data-sets indicated that organizational systems which 'Make doing the right thing easy' influences compliance, as does local ward culture. Embedded values and beliefs regarding the relative (de)-prioritization of MRSA screening are important.

Conclusion: To our knowledge, this is the first study to provide original evidence of barriers and enablers to MRSA screening, applying both sociological and psychological theory. As antimicrobial resistance is a global health concern, these findings have international relevance for screening programmes. Future policy recommendations or behaviour change interventions, based on the insights presented here, could have significant impact upon improving screening compliance. 


\section{Introduction}

The impact of screening for Meticillin resistant Staphylococcus aureus (MRSA) colonisation is evident in reported reductions in the rates of MRSA bacteraemia, which have been declining consistently since the introduction of mandatory MRSA surveillance and management policies [1-4]. Internationally, the cost-benefit of universal MRSA screening has been challenged $[5,6]$ and studies indicate that a targeted approach, including Clinical Risk Assessment (CRA) (where screening questions determine risk of colonisation and the need for microbiological swabs) and screening of patients admitted to high risk specialities (orthopaedics, intensive care, renal units), are more costeffective [6-9] particularly in areas of low MRSA prevalence. Additional modelling work conducted by Health Protection Scotland (HPS) identified that CRA of all admissions is as effective as universal screening when staff compliance with screening policy is $>90 \%$ [10]. This approach to MRSA screening has been adopted in Scotland, with a performance target of 90\% compliance with CRA set to maximise effectiveness [11].

National policies for MRSA admission screening are available [11] and the process may appear deceptively simple. However, what may appear straightforward from an infection prevention perspective may be considered a 'complex intervention' in the context of embedding screening into the everyday work of healthcare practitioners. Individual attitudes and beliefs, competing behaviours, as well as group dynamics and organisational contexts, may influence the actual implementation of screening intentions. These factors may shape compliance levels and the effectiveness of 'routine' screening practice in reducing the risk of transmission of MRSA. The national scale of this implementation challenge became apparent when audit data from self-selected areas submitted by all NHS Scotland Health Boards to HPS indicated a Scottish average MRSA screening compliance of $81 \%$ in 2015 [2].

In order to strengthen infection prevention, we must better understand those individual and group factors which may influence screening compliance. This can be achieved by utilising theoretical frameworks from the social sciences to study what helps or hinders the implementation of a complex intervention such as MRSA screening. Nilsen [12] categorises various theoretical frameworks used to explain influences on implementation outcomes, highlighting the importance of selecting an appropriate theoretical framework according to purpose; two different yet complementary theoretical frameworks seemed relevant here. Normalisation Process Theory (NPT) explores implementation from a sociological perspective. First developed by May and colleagues [13, 14], NPT looks at the social processes or 'work' undertaken by individuals and groups within an organisation to embed an intervention in routine practice (supplementary appendix 1). Within 
Nilsen's taxonomy, NPT has been categorised as an 'implementation theory', serving to provide causal explanations which enhance understanding of change mechanisms and the inter-relationships between constructs which influence intervention outcomes. Complementing this perspective, the Theoretical Domains Framework (TDF) $[15,16]$ adopts a more individualistic stance to understanding implementation by identifying factors that influence a person's decision to act or behave in specific situations (supplementary appendix 2). TDF is categorised by Nilsen as a 'determinants' framework, used to describe individual variables which may influence intervention outcome. However, TDF does not explore how change takes place nor highlight causal mechanisms for success or failure of implementation.

The aim of our study was to 'Provide evidence of barriers and enablers, offering an explanation of mechanisms that enhance or inhibit implementation of healthcare associated infection (HAI) screening policy in NHS hospitals.' As this aim encompassed both identification of individual barriers and enablers and explanation of causal mechanisms influencing implementation, the use of both TDF and NPT was warranted. The combination of these theoretical approaches in one study is relatively novel, although such pluralism is beginning to gain interest in intervention design [17] and evaluation [18].

\section{Methods}

A sequential 2-stage mixed methods design was used, applying constructs from NPT and the TDF to guide data collection and analysis. The use of such a 'theoretical lens' in developing data collection tools and forming a coding framework for data analysis and interpretation is recognised as valuable in strengthening the robustness of research, ensuring findings are theory driven[19, 20].

The study was reviewed and approved by the School of Health and Life Sciences Ethics Committee at Glasgow Caledonian University (HLS/NCH/15/18). Permission to access NHS staff was sought and granted from all 15 Scottish Health Board Executive Leads for healthcare associated infection. HPS acted as a gatekeeper to each NHS Board Infection Control Manager (ICM), who was then asked to forward study information and request voluntary participation from NHS staff on our behalf. All participants were provided with written study information and gave consent prior to participation.

\section{Stage 1}

\section{Data collection and analysis}

Stage 1 (February-March 2016) comprised qualitative telephone interviews and focus groups with clinical staff from four different Health Board sites, selected with advice from HPS for diversity in 
geographical location, hospital size, and MRSA CRA compliance (based on audit data from selfselected areas). Constructs from NPT and TDF were used to sensitise researchers to potential areas for exploration, which then helped shape the development of semi-structured, flexible topic guides (supplementary appendix 3), whilst allowing discussion to evolve as issues emerged from consecutive participants.

Data were collected via seven focus groups with ward based nursing staff (2-11 participants per group, total 38 nursing staff). The use of focus groups enabled participants to share their experiences working in diverse clinical areas and to compare and contrast approaches and challenges with MRSA screening. Focus groups began by asking participants to think, individually, about the process of MRSA screening and what helped or hindered them in complying with standard procedures. Participants then shared, in turn, their individual views and fuller group discussion of the range of experienced barriers and enablers ensued. This guided conversation highlighted the similarities and differences in implementation across different categories of clinical area. Individual interviews were conducted with Infection Control Managers (ICM) ( $n=4)$, Microbiology Leads $(n=4)$, and Bed Capacity Managers ( $n=3)$, representing each participating Health Board site. Interviews and focus groups were audio-recorded and transcribed verbatim.

Two approaches were used to analyse qualitative data. Firstly, transcripts were coded using principles from Braun and Clark's [21] approach to thematic analysis, resulting in two key themes presented below. Secondly, data were then coded into a framework consisting of NPT and TDF constructs to identify apparent barriers and enablers that were used in stage 2 of the study, to guide design of the survey tool. Rigour in data collection and analysis was maintained by peer review of coding by two researchers, with developing themes being discussed and agreed at team meetings; an audit trail of analytical decisions was maintained via NVivo $10^{\odot}$ software.

\section{Stage 1 Findings}

Two key themes emerged from initial qualitative analysis:

\section{Theme 1: 'It's the culture in there': the role of local culture in shaping staff beliefs}

Analysis suggested MRSA screening is enabled and constrained by local ward culture. Enabling cultures were associated with particular clinical contexts, namely pre-admission assessment clinics and locations implementing enhanced MRSA screening for high impact specialities (i.e. orthopaedics, renal medicine, and intensive care). In these locations screening was enabled by staff:

- fully understanding the purpose and importance of MRSA screening

- having clear leadership which prioritises MRSA screening as a key component of clinical care 
- perceiving MRSA as a serious risk with severe consequences for their patients

- taking MRSA screening very seriously (it is a priority within their work)

- having routinized processes that have embedded MRSA screening as an essential component of their work.

Cultures that constrained MRSA screening were also associated with particular clinical areas, for example, emergency receiving units. In these locations screening was constrained by staff:

- having such a high volume of patients and varied clinical demands that MRSA screening is lower on their priority list

- prioritising urgent clinical needs before MRSA screening

- being subject to senior management performance indicators which prioritise the need to maintain patient flow through the unit

- viewing the consequences of MRSA as experienced in 'down-stream' wards and therefore invisible.

Theme 2: 'Make doing the right thing easy': features of the immediate environment and hospital based systems that influence staff behaviour

Findings indicated that MRSA screening was enabled across all locations where there were systems in place providing triggers, prompts or cues to individuals. Enablers included:

- required CRA tool within the admission documentation, preferably appearing early on in paperwork (e.g. front page)

- mandatory (i.e. cannot complete admission without completing MRSA screening sections) CRA within an electronic admission system

- electronic patient alerts for previous MRSA positive status

- contact alerts from ICN for previous MRSA positive status

- regular contact with an ICN reminding / updating staff about CRA

- staff awareness that CRA compliance is audited and they receive compliance feedback.

Limitations in the availability of isolation facilities within the local environment were not reported to influence MRSA screening. However, lack of single rooms was reported to make the subsequent management of MRSA positive patients more challenging. Complex multidisciplinary decisions (involving IC Team, Senior Ward Staff, Bed Capacity Managers) are required to prioritise isolation facility allocation between patients with MRSA and other infections, as well as other clinical priorities such as the care of terminally ill patients. 
Summary tables of barriers and enablers aligned with respective NPT and TDF constructs are available in supplementary resources appendices 4 and 5 .

\section{Stage 2 Data collection and analysis}

Key barriers and enablers to MRSA screening elicited in stage 1 were used to develop questions for the Stage 2 national cross-sectional survey (supplementary appendix 6). Questionnaire items were mapped against relevant NPT and TDF constructs to enable interpretation of findings and subsequent explanation of mechanisms in action in light of relevant theories.

All 14 Scottish Health Boards and one Special Health Board were included in the survey $(n=15)$. A paper questionnaire was distributed via a hospital based link co-ordinator to 588 nursing staff in nine different types of clinical areas expected to undertake MRSA screening in acute care hospitals (general medical, renal, care of the elderly, pre-admission assessment clinic, emergency receiving/admission units (medical and surgical), general surgical, orthopaedic surgery and vascular surgery). These specialities were identified as the key areas for implementation of screening from Stage 1 of the study. Three paper-based questionnaires were distributed to each category of clinical area in each acute hospital: one to be completed by the nurse in charge and two by other nurses. Completed questionnaires were sealed, then collected and returned to the research team via the link co-ordinator.

Survey data was entered into SPSS (Version 23) ${ }^{\circledR}$ for analysis. Descriptive statistics and inferential testing (Chi-Square analyses) was used to explore the relationship between the dependent variable (self-reported compliance with MRSA screening) and the independent variables, to identify significant factors related to compliance for inclusion in a logistic regression model.

\section{Stage 2 Results}

The response rate for the paper-based survey for nursing staff was $86 \%$ (478/558). Due to some incomplete data, a final sample of 450 viable responses was included in descriptive analysis; 433 participants were included in the inferential analysis and modelling. Sample sizes met power calculation requirements (minimum sample size of 313 respondents) specifically for a logistic regression [22].

Nursing staff were asked 'When admitting patients to your clinical area, how many times do you manage to complete a MRSA clinical risk assessment?' (dependent variable). In keeping with the HPS 
target of over $90 \%$ compliance with CRA, 'optimum compliance' was subsequently defined as any self-report of ' $90 \%$ and above' compliance. Results are presented in Table I below, demonstrating that $76.2 \%(n=343)$ of respondents report optimum screening compliance, whilst $23.8 \%(n=107)$ of respondents do not normally achieve the national target.

\section{Insert here: Table I: Nursing staff self-reported compliance with MRSA CRA}

The following section focuses on the results of the logistic regression model; full descriptive and inferential results are available from the authors.

All significant variables (cut-off $p<0.05$ ) were entered into a multiple logistic regression model, which indicated three significant predictors for optimum compliance: the routinized nature of MRSA screening completion within the admission process ('MRSA screening is such a routine part of the admission process that I almost always complete it'); the provision of information relating to MRSA compliance within the recipients clinical area ('I am made aware of the level of compliance with MRSA screening achieved in the clinical area I work') and category of 'Clinical Area'. Tables II-IV present the results of the multiple regression model, separated into three tables for ease of reading, aligned with the respective theoretical constructs. Extracts of qualitative data are presented alongside each significant predictor in order to illuminate the findings.

\section{Insert here: Tables II-IV}

\section{Discussion}

There have been several published surveys which indicate limitations in the implementation of MRSA screening and management internationally; for example limited medical staff knowledge of MRSA infection control guidelines in the UK [25]; restricted implementation of national MRSA guidelines in Italy [26]; variable awareness of MRSA guidelines in an Egyptian hospital [27]. However, as far as we can ascertain, this is the first study to specifically investigate barriers and enablers to MRSA screening compliance at a national level, using sociological and psychological theory to interpret findings.

Our findings support HPS monitoring reports [2] which demonstrate that the national compliance target of $90 \%$ is not always achieved; $23.8 \%(n=107)$ of our respondents reported that they do not achieve optimum compliance levels. However, the aim of this study was to move beyond this suboptimal compliance statistic in order to identify those factors which present barriers and enablers to 
screening for MRSA in hospitals and to create an evidence base to develop interventions to improve compliance in the future.

Three key variables were found to predict self-reported optimum (>90\%) compliance with MRSA screening: having MRSA screening routinized within the admission process; the category of clinical area; feedback of MRSA screening compliance within the clinical area. The following discussion explores each of these key predictors in turn, utilising both NPT and TDF to explain possible mechanisms which help or hinder implementation and barriers or enablers to appropriate compliance behaviour. Links with comparable findings from other studies are also outlined.

The most important variable in terms of its relative contribution to explaining optimal MRSA screening behaviours related to agreement with the routinized nature of MRSA within the admission process. This finding signals the importance of organisational systems and processes that have become normalised and embedded in practice. In this way, MRSA screening is facilitated through routinized habits and behaviours that do not require individual level decision-making. Subsequently, implementing these behaviours requires little reflective thought or decision-making for front line staff. In NPT terms, routinized admission MRSA screening is coherent; participants understand what MRSA screening requires of them; and they enact the work of screening by performing the tasks required. From a TDF perspective, the environmental context provides an organisational system which presents reinforcement by way of aide memoires which prompt staff to screen. By facilitating routine and habit within the admissions process, optimal screening is more easily enabled. Equally, this suggests that where screening is not routinized within the admissions process, key barriers to screening remain. These barriers and enablers are illustrated in the qualitative data presented in table II, indicative of the Stage 1 theme 'Make doing the right thing easy'.

The clinical area that participants work in also made a major relative contribution to understanding variance in optimal MRSA CRA screening behaviour. The variable 'category of clinical area' reflects the influence of the local ward culture beyond other measured variables associated with organisational systems intended to promote screening, such as admissions procedures. Defined as "values and behaviours that contribute to the unique social and psychological environment of an organization", culture is based on "shared attitudes, beliefs, customs, and written and unwritten rules that have been developed over time and are considered valid"[28]; variation in the ward culture between different types of clinical area was apparent in our findings.

This cultural dimension taps into the range of very different demands and norms relating to implementing screening associated with specific clinical areas. NPT would consider the relative work 
undertaken by participants to normalise MRSA screening in situations where the potential value and prioritisation of CRA is appraised differently according to category of clinical area. Our findings indicate the importance of pressures from other work and the relative perceived importance of MRSA screening compared to other activities and management priorities. NPT explains these mechanisms as related to coherence, where staff groups internalise the value of MRSA screening and thus participation in screening is normalised where they and their colleagues are led to believe it is an important part of their work; the converse also holds true. Similarly, TDF highlights the function of social influences which operate differentially across clinical areas. These social influences determine relative risk perception, beliefs about consequences, and professional role beliefs, and either enable or inhibit individual actions towards compliance with screening. The influence of different clinical areas in the implementation of MRSA protocols was noted as far back as 2005, where work by van Gemert-Pijnen et al. [29] demonstrated that whilst staff knowledge of and attitudes towards MRSA protocols was adequate, implementation in different workplaces was inconsistent. Based on these earlier findings, in discussing their survey of Egyptian staff awareness of MRSA guidelines, Soliman et al [27] suggest that tailored MRSA protocols should be developed which take account of the varying priorities and pressures within different departments. Our findings would support this recommendation for a more tailored approach to guideline or intervention implementation, which acknowledges of the different nature and challenges experienced in different clinical areas; recognising that 'It's the culture in there' that can influence screening compliance.

The third important variable explaining optimal MRSA screening behaviour is whether participants agreed with the following statement 'I am made aware of the level of compliance with MRSA screening achieved in the clinical area I work'. This item again captures a particular dynamic linking the local organisation of services to the individual's propensity to implement MRSA screening. The finding suggests that when systems are in place that provide feedback on behaviour, screening is enabled. This feedback mechanism relates to the NPT construct of systemisation of appraisal, where participants are able to access feedback on their performance, as well as the TDF domain of behavioural regulation, where organisational systems, generally operationalized by the IPC team, are in place to provide feedback on screening compliance. Qualitative data from our study (Table IV) indicates the importance of the role of the Infection Control Team (ICT) and the resources at their disposal, which can actively facilitate this feedback in a constructive way. This finding is endorsed by Ward [30], who argues that Infection Control Nurse's should act as leaders to help motivate nurses to engage with IPC practices. Conversely, Ward's interview based study of nurses and nursing students ( $n=63$ ) working in one region found that the IPC Nurse was often viewed with fear and as a threat, someone who visited to criticise; "you are doing that wrong". The importance of the IPC 
Nurse having a regular presence, rather than visiting only when problems arose, and having an approachable attitude was emphasised here [30]. The value of this collegiate approach was evident in much of our data; however, ICTs in some NHS Boards reported not having sufficient resources to enable the IPC Nurse to function in this way (Table IV). Thus the available resource and model of working of ICTs may influence the provision of constructive feedback, which would in turn influence screening compliance.

We acknowledge limitations in this study and that other factors, not assessed here, are potentially influential in optimal compliance with MRSA screening. A further limitation of the statistical analysis is that the dependant variable is self-reported compliance with screening, which cannot be independently validated.

A key strength of this research is the mixed methods design, with the qualitative elicitation of participant generated factors used to inform the development of the quantitative survey tool. The use of NPT and TDF to sensitise researchers during data collection and analysis, whilst remaining open to more grounded data gathering during qualitative stages, is a relatively novel methodological approach which adds theoretical rigour and explanatory potential to the study. The conduct of a multi-site, nationally distributed survey also adds weight to the findings.

\section{Recommendations}

The findings from this study make an original contribution to the evidence base concerning the implementation and embedding of MRSA screening and a series of preliminary recommendations are possible.

Changes to address organisational components such as routinizing admission screening and feedback of compliance data should be considered. For instance, patient management systems which incorporate recording of MRSA screening within the admission process should be strengthened, possibly by the use of electronic patient information systems which provide clear instruction and prompt staff to complete screening. Feedback provided directly to staff can influence behaviour, therefore national and local screening compliance monitoring systems should ensure feedback loops are built into the process, as this appears inconsistent at present. Our data indicates the value of Infection Control Team (ICT) interaction with and support of clinical staff in screening and feedback of compliance results, although ICT teams report operating in different ways across the country. Thus, the availability of ICT resource to undertake this type of engagement activity may require further review. 
Generating solutions to the challenge of local ward cultures where there is relative de-prioritisation of MRSA screening in the face of competing clinical priorities and work pressures is more problematic. Further research to explore the processes of (de)-prioritisation of screening within different clinical contexts is advisable. Policy and practice stakeholder debate regarding the resource requirements to enable effective screening for multi-drug resistant organisms, particularly in emergency admission receiving areas, is recommended. Additional implementation studies to design and evaluate the impact of tailored interventions within specific categories of clinical area are warranted.

\section{Conclusions}

This study provides original evidence of barriers and enablers to MRSA screening from the perspectives of nursing staff from a range of clinical areas across all NHS Scotland Boards.

Integration of qualitative findings and logistic regression modelling indicates that having routinized admission systems in place, associated with monitoring and feedback of screening compliance data enables optimum compliance; where organisations 'make doing the right thing easy'. Conversely, even when these systems are apparent within the organisation, local ward culture and practice can present either barriers or enablers to optimum compliance, with embedded values and beliefs regarding the relative (de)-prioritization of CRA being influential; inherently, 'It's the culture in there' that also impacts on screening behaviours and tailored approaches to screening guideline implementation are required.

The challenges of overcoming barriers to compliance whilst maximising the impact of those factors which enable optimum compliance are significant. However, the use of the combined theoretical frameworks applied in this study provides greater depth of understanding of potential mechanisms of successful implementation and should be utilised to direct future interventions.

\section{References}

[1] Centers for Disease Control and Prevention. (2017). Healthcare-associated Infections in the United States, 2006-2016: A Story of Progress. Retrieved from https://www.cdc.gov/hai/surveillance/data-reports/data-summary-assessing-progress.html

[2] Health Protection Scotland. (2017). Scottish One Health Antimicrobial Use and Antimicrobial Resistance Report 2016.2 Retrieved from Glasgow: http://www.hps.scot.nhs.uk/pubs/detail.aspx?id=3378

[3] Kock R, Becker K, Cookson B et al. Systematic literature analysis and review of targeted preventive measures to limit healthcare-associated infections by meticillin-resistant Staphylococcus aureus. Euro Surveill 2014; 19. 
[4] Public Health England. (2017). Annual Epidemiological Commentary Mandatory MRSA, MSSA and E. coli bacteraemia and C. difficile infection data 2016/17. Retrieved from London: https://www.gov.uk/government/uploads/system/uploads/attachment data/file/634675/A nnual epidemiological commentary 2017.pdf

[5] McKinnell JA, Bartsch SM, Lee BY, Huang SS, Miller LG Cost-Benefit Analysis from the Hospital Perspective of Universal Active Screening Followed by Contact Precautions for Methicillin-resistant Staphylococcus aureus Carriers. Infect Control Hosp Epidemiol 2015; 36: 2-13.

[6] Roth VR, Longpre T, Coyle D et al. Cost Analysis of Universal Screening vs. Risk Factor-Based Screening for Methicillin-Resistant Staphylococcus aureus (MRSA). PLOS ONE 2016; 11: e0159667.

[7] Tübbicke A, Hübner C, Hübner N-O, Wegner C, Kramer A, Fleßa S Cost comparison of MRSA screening and management - a decision tree analysis. BMC Health Serv Res 2012; 12: 438-.

[8] Deeny SR, Cooper BS, Cookson B, Hopkins S, Robotham JV Targeted versus universal screening and decolonization to reduce healthcare-associated meticillin-resistant Staphylococcus aureus infection. J Hosp Infect 2013; 85: 33-44.

[9] Cairns S, Packer S, Reilly J, Leanord A Targeted MRSA screening can be as effective as universal screening. BMJ : British Medical Journal 2014; 349.

[10] Health Protection Scotland. (2011). NHS Scotland MRSA Screening Pathfinder Programme: The Value of Nasal Swabbing versus Full Body Screening or Clinical Risk Assessment to Detect MRSA Colonisation at Admission to Hospital. Retrieved from Glasgow: http://www.hps.scot.nhs.uk/resourcedocument.aspx?id=2496

[11] Health Protection Scotland. (2013). Protocol for CRA MRSA Screening National Rollout in Scotland, Version: 1.7. Retrieved from Glasgow: http://www.hps.scot.nhs.uk/resourcedocument.aspx?resourceid=1639

[12] Nilsen P Making sense of implementation theories, models and frameworks. Implementation Science : IS 2015; 10: 53.

[13] May C, Finch T, Mair F et al. Understanding the implementation of complex interventions in health care: the normalization process model. BMC Health Serv Res 2007; 7: 148.

[14] May C, Rapley T, Mair FS et al. Normalization Process Theory On-line Users' Manual, Toolkit and NoMAD instrument. . http://www.normalizationprocess.org/ (02 Feb 2018 2018, date last accessed).

[15] Francis JJ, O'Connor D, Curran J Theories of behaviour change synthesised into a set of theoretical groupings: introducing a thematic series on the theoretical domains framework. Implementation Science 2012; 7: 35.

[16] Cane J, O'Connor D, Michie S Validation of the theoretical domains framework for use in behaviour change and implementation research. Implementation Science 2012; 7: 37.

[17] Band R, Bradbury K, Morton $\mathrm{K}$ et al. Intervention planning for a digital intervention for selfmanagement of hypertension: a theory-, evidence- and person-based approach. Implementation Science 2017; 12: 25.

[18] Backman R, Weber P, Turner AM, Lee M, Litchfield I Assessing the extent of drug interactions among patients with multimorbidity in primary and secondary care in the West Midlands (UK): a study protocol for the Mixed Methods Multimorbidity Study (MiMMS). BMJ Open 2017; 7.

[19] Stewart D, Klein S The use of theory in research. Int J Clin Pharm 2016; 38: 615-9.

[20] Bradbury-Jones C, Taylor J, Herber O How theory is used and articulated in qualitative research: development of a new typology. Soc Sci Med 2014; 120: 135-41.

[21] Braun V, Clarke V Using thematic analysis in psychology. Qualitative Research in Psychology 2006; 3: 77-101.

[22] Peduzzi P, Concato J, Kemper E, Holford TR, Feinstein AR A simulation study of the number of events per variable in logistic regression analysis. J Clin Epidemiol 1996; 49: 1373-9. 
[23] Cole TJ Too many digits: the presentation of numerical data. Arch Dis Child 2015; 100: 608-9.

[24] Cole TJ Setting number of decimal places for reporting risk ratios: rule of four. BMJ : British Medical Journal 2015; 350.

[25] Brady RR, McDermott C, Cameron F, Graham C, Gibb AP UK healthcare workers' knowledge of meticillin-resistant Staphylococcus aureus practice guidelines; a questionnaire study. $J$ Hosp Infect 2009; 73: 264-70.

[26] Pan A, Bombana E, Tura G et al. A survey of methicillin-resistant Staphylococcus aureus control strategies in Italy. Infection 2013; 41: 783-9.

[27] Soliman GS, Abu-Youssef RM, Saleib BF, El-Moughazi AM, Zaki A Awareness of World Health Organization methicillin-resistant Staphylococcus aureus guidelines at Alexandria University hospitals. East Mediterr Health Journal 2013; 19: 622-8.

[28] WebFinance Inc. Business Dictionary, http://www.businessdictionary.com/definition/organizational-culture.html (June 2018.

[29] van Gemert-Pijnen J, Hendrix MGR, van der Palen J, Schellens PJ Performance of methicillinresistant <em $>$ Staphylococcus aureus $</$ em $>$ protocols in Dutch hospitals. Am J Infect Control; 33: 377-84.

[30] Ward DJ Attitudes towards the Infection Prevention and Control Nurse: an interview study. J Nurs Manag 2012; 20: 648-58. 